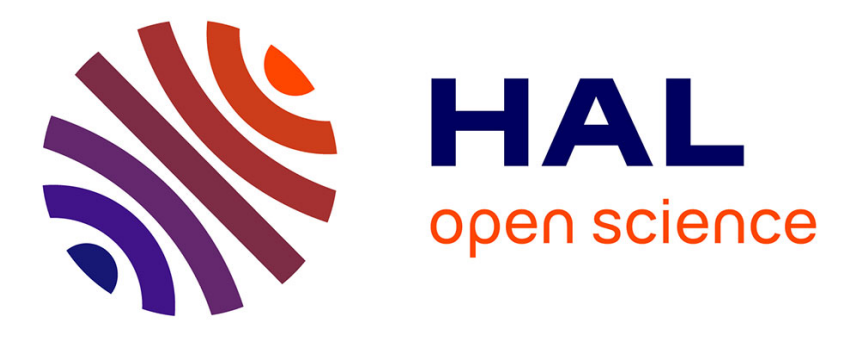

\title{
Façonnement du corps vieillissant par les technologies
}

Lucie Dalibert

\section{To cite this version:}

Lucie Dalibert. Façonnement du corps vieillissant par les technologies. Gérontologie et Société, 2015, Regards croisés sur le corps vieillissant, 37 (148), pp.47-58. 10.3917/gs1.148.0047 . halshs$01650113 \mathrm{v} 2$

\section{HAL Id: halshs-01650113 \\ https://shs.hal.science/halshs-01650113v2}

Submitted on 14 Feb 2020

HAL is a multi-disciplinary open access archive for the deposit and dissemination of scientific research documents, whether they are published or not. The documents may come from teaching and research institutions in France or abroad, or from public or private research centers.
L'archive ouverte pluridisciplinaire HAL, est destinée au dépôt et à la diffusion de documents scientifiques de niveau recherche, publiés ou non, émanant des établissements d'enseignement et de recherche français ou étrangers, des laboratoires publics ou privés. 


\section{Lucie Dalibert}

Façonnement du corps vieillissant par les technologies

Le vieillissement est rarement appréhendé comme allant de pair avec les technologies. Cet article discute comment une technologie implantée, la stimulation de la moelle épinière, construit le vieillissement et constitue le corps âgé. C'est à travers l'exploration de l'incarnation et de l'incorporation de cette technologie, laquelle repose sur une approche postphénoménologique du corps et des technologies et s'appuie sur 18 entretiens réalisés au printemps 2012 aux Pays-Bas avec des personnes vivant avec cette technologie, un neurochirurgien, une infirmière et un développeur de produits, que cette problématique est abordée. Alors que le corps vieillissant devient un corps en pièces, le vieillissement est clé pour pouvoir bien vivre avec des technologies implantées.

Mots clés : Vieillissement ; Corps ; Technologies ; Incarnation ; Incorporation

The shaping of the aging body by technologies

Aging is rarely apprehended as going hand in hand with technology. This article discusses how an implanted technology, spinal cord stimulation, constructs aging and the aged body. It is through the exploration of the technology's embodiment and incorporation, which is grounded in a post-phenomenological approach to bodies and technologies and which is based on 18 interviews conducted in spring 2012 in the Netherlands with people living with the technology, a neurosurgeon, a nurse and a product developer, that this issue is addressed. While the aging body becomes a body in parts, aging is key to living well with implanted technologies.

Keywords: Aging; Bodies; Technologies; Embodiment; Incorporation 
Alors que le vieillissement de la population soulève des inquiétudes quant à la viabilité des systèmes de santé et des régimes de retraite ainsi qu'à la capacité d'innovation des économies et la solidarité entre générations, les innovations technologiques sont proposées comme solution afin que le vieillissement soit « actif et en bonne santé » (Peine et al., 2015). Le corps vieillissant est devenu un lieu privilégié d'interventions technoscientifiques (Joyce et Mamo, 2006).

Or, les corps, et plus encore les corps vieillissants ont tendance à être négligés dans les travaux issus de la philosophie des technologies et des études des sciences et des technologies (Science and Technology Studies, STS) dont l'objet porte sur les relations entre les êtres humains et les technologies. Ceux-ci ont exposé la biomédicalisation croissante du vieillissement, à savoir son appréhension comme un problème qui exige une intervention biomédicale (Estes et Binney, 1989 ; Kaufman et al., 2004), ainsi que les représentations négatives et préjudiciables (âgistes) de la vieillesse qui peuvent sous-tendre la conception des technologies (Neven, 2011). Ils ont aussi montré que les technologies visant à ce que les personnes âgées restent actives et autonomes à domicile peuvent se révéler coercitives et conduire à des injonctions contradictoires sur ce que le « bien vieillir » se doit d'être (Mort et al., 2013). Ces études ont également indiqué que les personnes âgées ne sont pas des consommatrices passives, réticentes ou ignorantes de produits technologiques, mais qu'elles se les approprient et les utilisent de façon créative dans leur vie quotidienne : elles sont des « technogénaires » (Joyce et Loe, 2010). De surcroît, ces analyses ont souligné que ce sont les personnes âgées qui ont tendance à vivre intimement avec les technologies, invitant ainsi à « griser le cyborg » (Joyce et Mamo, 2006).

Les études en philosophie des technologies et STS sur les technologies et le vieillissement n'ont que peu suivi cette invitation, laissant le corps vieillissant de côté. Lorsqu'elles se sont portées sur les corps « cyborgs », lesquels matérialisent l'intimité des 
relations entre les êtres humains et la technologie, elles ont montré que les technologies (prothétiques et implantées) sont conçues comme venant perfectionner des corps jeunes et valides ; si des corps amputés et/ou handicapés - mais jamais vieux - apparaissent, ce n'est qu'en tant que symboles d'une existence cyborg mais pas pour la singularité de leurs expériences avec ces technologies (Betcher, 2001 ; Dalibert, 2015 ; Haraway 1991 ; Maestrutti, 2011 ; Sobchack, 2006). Outre que le vieillissement est forclos de l'imaginaire cyborg - corps âgés et corps cyborgs apparaissent comme antithétiques - très peu de travaux se sont penchés sur les pratiques et les expériences des personnes vivant avec des technologies implantées et prothétiques, lesquelles tendent à constituer le corps cyborg certes, mais aussi et de plus en plus le quotidien des personnes âgées (Kaufman et al., 2011). De même, si certaines études (p. ex. Gérardin et Andrieu, 2011 ; Macia et al., 2013) signalent l'« hybridation » croissante à laquelle sont confrontées ces dernières, le corps tel qu'il est transformé par divers prothèses et implants et tel qu'il est alors vécu reste inexploré.

Sur cette toile de fond, cet article interroge le rôle des technologies dans le vieillissement, et plus particulièrement le façonnement du corps vieillissant (et son vécu) par les technologies implantées. C'est à la croisée de la philosophie des technologies et des STS, et en nous appuyant sur un travail de terrain réalisé aux Pays-Bas dans le champ des technologies de neuromodulation que nous aborderons cette question. Après un cadrage théorique et méthodologique, nous expliquerons comment l'on vit avec une technologie de stimulation de la moelle épinière et nous montrerons dans quelle mesure le vieillissement est clé pour comprendre pourquoi cette technologie peut être bien ou mal vécue. Pour ce faire, nous convoquerons les concepts d'incarnation et d'incorporation issus des approches postphénoménologiques de la technologie. 


\section{1. Étudier les relations intimes entre êtres humains et technologies}

Loin du vieillissement, la recherche qui sous-tend cet article a porté sur les relations intimes entre les êtres humains et les technologies en lien avec l' " augmentation humaine » (Dalibert, 2014). Toutefois, le vieillissement y est apparu comme clé pour comprendre certaines facettes de ces relations. L'une des technologies étudiées au cours de cette recherche en philosophie empirique $^{1}(\mathrm{Mol}, 2002)$ et sur laquelle cet article repose est une technologie de neuromodulation : implantée, la stimulation de la moelle épinière est utilisée comme traitement de dernier recours contre la douleur chronique. Dans la mesure où ce sont les expériences des personnes qui vivent avec cette technologie qui nous ont intéressée, nous avons adopté une méthodologie qualitative et une approche post-phénoménologique des technologies.

Partant de M. Merleau-Ponty qui a montré que « le corps est notre moyen général d'avoir un monde » (1945, p. 171), cette approche reconnaît non seulement le caractère situé et incarné des êtres humains mais aussi « l'agentialité » des objets technologiques. Ceux-ci ne sont pas des instruments neutres ou passifs : ils matérialisent des normes et des valeurs.

Comme l'ont exposé M. Akrich (1992) et B. Latour (1992), les objets technologiques ont un «script » qui prescrit certaines actions, comme le fait un film. Ces scripts invitent certaines actions et en découragent d'autres. Ainsi, les technologies transforment nos actions, nos perceptions et nos identités, et peuvent être utilisées de manière inattendue et créative (Oudshoorn et Pinch 2003). Dès lors, les êtres humains n'ont pas un accès direct au monde, mais via les technologies qui l'habitent et le constituent : les philosophes D. Ihde (1990) et P.P. Verbeek (2005) parlent de « médiation technologique ». Ihde en identifie deux instances : les relations herméneutiques et d'incarnation (embodiment). Dans les premières, le monde est

\footnotetext{
${ }^{1}$ La philosophie empirique (Mol, 2002) incorpore réflexion philosophique et matériel empirique (généralement recueilli à l'aide d'entretiens et d'observations). Elle fait écho au «tournant empirique » de la philosophie des technologies dans lequel cet article est ancré et qui préfère l'exploration des objets technologies dans leurs relations concrètes avec les êtres humains à une conception transcendantale de la Technologie (Verbeek, 2005).
} 
perçu au moyen de l'objet technologique (p. ex. un thermomètre ou un radiotélescope), lequel fournit une vision du monde qui doit être interprétée. Dans les relations d'incarnation, le monde est vécu à travers l'objet technologique (p. ex. des lunettes ou une canne) : si l'objet étend le champ de perception, il est perçu comme un « quasi-moi ». N'attirant pas (ou plus) l'attention, il devient (quasi) « transparent » aux niveaux sensoriel et cinétique, il est incarné dans le « schéma corporel » (Merleau-Ponty, 1945). Cette configuration, qui dénote l'intimité des relations entre êtres humains et technologies, est centrale dans les relations entre les personnes vivant avec la stimulation de la moelle épinière et celle-ci.

Bien qu'elle soit l'état désiré - et l'indication d'un usage réussi - de l'objet technologique dans les relations d'incarnation, la « transparence » n'est pas immédiate mais plutôt le résultat d'un processus dans lequel le corps, ses gestes et ses postures sont fondamentaux. Comme l'a décrit C. Noland dans ses travaux qui explorent comment le corps incarne ou résiste aux forces culturelles, c'est à travers un processus de «tâtonnement »-elle reprend le concept au paléoanthropologue A. Leroi-Gourhan - que le corps change et s'habitue à de nouvelles influences. Comme elle l'explique, « tâtonner ${ }^{2}$ traduit l'exploration, qu'elle soit physique ou cognitive : tester un chemin qui n'est pas encore défriché ou concevoir un ordre qui n'est pas encore établi » (Noland, 2009, p. 105-106). C'est à l'issue de tels expérimentation et apprentissage que progressivement la technologie est vécue de manière (quasi) transparente. Pour autant nécessaire afin que l'objet technologique ne soit pas ressenti comme une gêne, le processus d'incarnation n'est pas suffisant pour expliquer comment il peut être bien ou mal vécu. Bien qu'incarné - perçu comme un « quasi moi » aux niveaux sensoriel et cinétique - l'objet technologique peut toujours être vécu comme étrange(r) au corps - à « l'image de soi » - et être la cause de souffrances.

\footnotetext{
${ }^{2}$ En français dans le texte.
} 
À cet égard, au sein des approches post-phénoménologiques de la technologie, quelques travaux qui se sont intéressés aux transformations corporelles induites par les objets technologiques - les implants et les prothèses en particulier, mais aussi les transplantations ont mis l'accent sur le processus d'incorporation et ont exposé son importance dans l'acceptation de la technologie et des changements corporels qu'elle engendre. L'incorporation renvoie à la possibilité et à la capacité de concevoir l'objet technologique comme faisant partie de son corps, tel que peut l'être sa propre main ou son propre pied. Si certains ont lié l'incorporation d'un objet étrange(r) à un « sentiment de propriété du corps » (De Preester, 2011), d'autres ont montré que pour que cet objet - une main greffée p. ex. soit vécu comme faisant partie de son corps, c.-à-d. pour qu'il soit intégré à « l'image de soi », il faut pouvoir s'identifier avec son corps nouvellement transformé (Slatman et Widdershoven, 2010 ; voir aussi Sobchack, 2010). Plus précisément, J. Slatman et G. Widdershoven (2010) ont montré que l'identification au corps transformé n'est pas seulement visuelle mais également affective et tactile, et elle engage le corps des autres, qu'il s'agisse du corps d'êtres aimés, de celui plus lointain mais non moins marquant de passants inconnus, ou encore du corps social et son univers normatif.

Dans le cadre de notre recherche exploratoire sur les relations intimes entre les êtres humains et les technologies, au printemps 2012 nous avons réalisé des entretiens semistructurés (en anglais) avec quinze personnes vivant avec la stimulation de la moelle épinière (le conjoint ou l'un/e des enfants étaient parfois présents) et deux autres avec l'infirmière et le neurochirurgien qui s'occupent de ces personnes à l'hôpital où nous avons été autorisée à effectuer notre travail de terrain. Nous avons aussi effectué un entretien avec un ancien développeur de produits pour St Jude Medical, l'entreprise qui développe la technologie de neuromodulation étudiée, et nous avons eu des contacts continus avec une physicienne médicale qui fut notre informatrice principale dans ce terrain. Nous avons également observé 
des procédures d'implantation et des visites de contrôle. Cette recherche a été approuvée par le comité d'éthique de l'hôpital, et toutes les personnes interrogées ont verbalement donné leur consentement à y participer. Pour assurer leur anonymat, nous utilisons des pseudonymes.

Créant un espace pour exprimer leur ressenti sur les changements apportés par la technologie, ces entretiens nous ont permis de recueillir les expériences des personnes qui vivent avec la stimulation de la moelle épinière. En les relisant et les recoupant, nous avons élaboré une grille de lecture pour notre analyse, que nous avons raffinée jusqu'à ce qu'elle soit suffisamment compréhensive (Laz, 2003). Bien que les entretiens réalisés soient relativement peu nombreux, l'utilisation de cette grille nous a permis d'identifier des tendances communes ou singulières aux répondants. Celles-ci sont présentées ici, notamment à travers la reconstruction des récits de Mme Bloemen et M. van Houten.

\section{Vivre avec la stimulation de la moelle épinière}

Mme Bloemen (45 ans) et M. van Houten (61 ans) vivent avec la technologie de neuromodulation depuis 2009 du fait de douleur chronique due à une neuropathie diabétique pour elle et à une lombalgie chronique pour lui. La stimulation de la moelle épinière est utilisée comme traitement de dernier recours contre la douleur chronique causée par ces deux conditions. Implantée, cette technologie agit directement sur les tissus nerveux de la moelle épinière : elle en module l'activité. Plus précisément, l'administration continue d'impulsions électriques sur les colonnes dorsales de la moelle épinière interrompt les signaux de douleur, réduisant ainsi sa perception (dans le dos, les jambes et les pieds). Ce faisant, la stimulation de la moelle épinière ne guérit pas la cause de la douleur, mais remplace sa perception par une autre sensation «plus agréable » appelée paresthésie, décrite généralement comme une sensation de picotement. 
Pendant l'opération réalisée sous anesthésie locale durant laquelle le/la patient/e est allongé/e sur le ventre, le neurochirurgien place, à l'aide de clichés radiographiques, une électrode mesurant 6 centimètres de long, 5 millimètres de large et se composant de 8 contacts dans l'espace épidural. Ensuite, il relie l'électrode à un neurostimulateur (situé à l'extérieur du corps) que l'infirmière commence alors à programmer. Les paramètres de stimulation sont définis et ajustés en coordination avec le/la patient/e. Éveillé/e, il/elle indique si la colonne dorsale est stimulée ou non au bon endroit avec la bonne intensité, le champ électrique généré sur la colonne dorsale devant exciter les fibres qui innervent la zone douloureuse et la paresthésie provoquée par la stimulation devant la couvrir complètement. Une fois le programme de stimulation défini, l'opération peut se terminer. Après une nuit à l'hôpital, les patients rentrent chez eux. S'ensuit une période d'essai de plusieurs jours : ce n'est que si un soulagement significatif de la douleur est ressenti que le neurostimulateur externe est converti en un plus petit qui est implanté sous la peau (en bas de l'abdomen ou en haut des fesses) lors d'une deuxième intervention chirurgicale. Aux Pays-Bas, la plupart des neurostimulateurs implantés sont non-rechargeables et doivent être remplacés chirurgicalement après quelques années.

En plus de l'électrode et du neurostimulateur, le système de stimulation de la moelle épinière compte des composants externes : une télécommande et une antenne ${ }^{3}$. Pour être utilisée, la télécommande doit être reliée à l'antenne, elle-même positionnée sur le neurostimulateur (situé sous la peau du bas-ventre ou en haut des fesses) : neurostimulateur et télécommande sont ainsi connectés par fréquence radio. Bien que l'on puisse utiliser la télécommande pour choisir entre différents programmes et modifier l'intensité de la stimulation quand la douleur varie, on ne peut pas modifier ni redéfinir ses paramètres : cela ne peut être fait par un/e infirmièr/e spécialisé/e lors des visites de contrôle. Pour la plupart

\footnotetext{
${ }^{3}$ Voir http://www.medgadget.com/2006/05/eon rechargeabl.html [visité le 20 février 2015].
} 
des personnes vivant avec la stimulation de la moelle épinière, la procédure d'implantation est une expérience très exigeante, pour ne pas dire traumatique. Mais le rôle essentiel voire vital joué par cette technologie dans le soulagement de la douleur fait oublier, chez toutes les personnes interrogées, cet événement perturbant. L'implantation de la technologie est en fait vécue comme une « renaissance » :

Ça a changé ma vie. Ce que je peux faire et ce que je ressens. ... Sans ça, je vis dans beaucoup de douleur, je suis toujours couché dans mon lit ou bien assis dans un fauteuil roulant, toute la journée. Je suis assis et je regarde dehors, je ne fais rien - peut-être, oui, prendre un café à la maison. Mais maintenant, je peux aller à l'extérieur, je peux aller faire des courses, je peux prendre soin de mon mari. Et quand je n'ai pas ça [la technologie de neuromodulation], [soupir] oui, alors je ne peux rien faire. ... Maintenant je peux dire que je suis de nouveau quelque part. Je fais maintenant partie de la vie (M. van Houten) ${ }^{4}$.

M. van Houten apprécie grandement la technologie de neuromodulation qui lui permet d'agir et de faire à nouveau partie du monde ou, comme il le dit, de la vie. Il en est si content que, comme il nous l'explique plus tard, cette technologie fait partie de lui et il aimerait qu'une fermeture éclair soit placée dans son dos afin que tout le monde puisse voir l'électrode qu'il appelle un pacemaker pour ses jambes et son dos. Cette reconnaissance et ce soulagement engendrés par la technologie ne sont toutefois pas directs, ni instantanés, ni ressentis par toutes les personnes qui vivent avec la stimulation de la moelle épinière.

À cet égard, bien que Mme Bloemen reconnaisse que celle-ci est une aide précieuse pour minimiser sa douleur chronique et qu'elle a changé sa vie, lui permettant d'aller se promener ou de faire les magasins avec sa fille, elle n'en demeure pas moins insatisfaite et même malheureuse vis-à-vis de cette même technologie. Outre sa frustration liée au fait que

\footnotetext{
${ }^{4}$ Les citations (entretiens et ouvrages) ont été traduites par l'auteur.
} 
l'écran de la télécommande est trop étroit pour qu'elle puisse lire ce qui y est indiqué et que ses boutons sont trop petits pour qu'elle puisse la manier, elle est particulièrement affectée par la technologie de neuromodulation dans la mesure où elle la considère comme ne faisant pas partie de son corps, de son « image de soi ». Elle ne peut ni toucher le bas de son dos ni le regarder dans le miroir sans sentir et voir (sous la peau) la forme du neurostimulateur; elle est d'autant plus désolée que son mari puisse aussi le voir et le toucher.

Les expériences de M. van Houten et Mme Blomen avec la stimulation de la moelle épinière indiquent que vivre avec une telle technologie n'est pas une simple affaire, mais comment expliquer ces différents ressentis? Pour cela, il est nécessaire d'analyser ce que cette technologie fait aux corps.

\section{Corps en mouvement et technologie : l'incarnation comme tâtonnement}

Si la stimulation de la moelle épinière soulage la douleur chronique, la remplaçant par une sensation «plus agréable» de picotement, elle requiert un certain apprentissage. Avoir recours à la télécommande pour moduler l'intensité de la stimulation ou pour changer de programme est ce que l'on pourrait appeler le comportement «prescrit » par la technologie de neuromodulation (Akrich, 1992 ; Latour, 1992). Or, la télécommande n’est pas nécessairement centrale dans les relations que l'on peut avoir avec la stimulation de la moelle épinière.

Mme Jansen (63 ans), qui vit avec la technologie de neuromodulation depuis 2005, explique en ces termes comment la stimulation de la moelle épinière fonctionne pour elle et ce qu'elle ressent :

Et bien, on le sent directement [..] C'est comme ça que je le ressens à l'instant. [..]

La sensation passe dans les jambes et le dos ici [..] Je le sens. Et puis, si je suis allongée, ou si je mets ma tête en arrière, je le sens très clairement. [..] Si mes 
jambes sont douloureuses, je fais ça [elle bouge sa tête vers l'arrière], alors ça vibre très fortement pendant un moment et la douleur diminue.

La technologie de neuromodulation crée une sensation distincte, laquelle peut être dirigée par la réalisation de certains gestes et postures. Si ces mouvements corporels permettent d'établir et d'entretenir une relation (intime) avec la technologie, ils sont aussi essentiels pour que celle-ci et ses effets - paresthésie - n'absorbent ni l'attention ni ne soient vécus comme gênants, mais plutôt pour que la technologie de stimulation de la moelle épinière devienne (quasi) transparente, c.-à-d. pour qu'elle soit incarnée (Ihde, 1990).

En effet, la disparition de la technologie de neuromodulation sous la peau n'équivaut pas à une transparence de facto. Implantation ne veut pas dire incarnation - ni incorporation. L'incarnation de la technologie, qui implique l'habituation à de nouvelles sensations, résulte d'un certain « tâtonnement ». M. van Houten décrit ainsi les premiers jours, voire les premières semaines qui suivent l'implantation :

[L]es premiers deux, trois jours, oui, oui, toute la journée vous êtes juste à jouer avec ça [la technologie]. Oui. Qu'est-ce qui est possible ? Est-ce que je peux conduire la voiture ? [..] Lorsque vous êtes assis : oh, c'est très agréable. Et puis vous allez vous lever : iuuuuuh ! Oui ?... Vous augmentez l'intensité : ah, ce n'est pas possible ! Et puis vous devez apprendre : plus fort, que pouvez-vous faire ? Je fais des essais : qu'est-ce je sens quand c'est plus ou moins fort? Faire varier l'intensité de la stimulation et en évaluer cinétiquement l'effet, faire certains gestes et mouvements, adopter certaines postures et en évaluer l'effet en termes de stimulation : c'est à travers ce tâtonnement que progressivement la technologie devient transparente. Ce devenir-transparent de la technologie, Mme Jansen le décrit en ces termes :

Au début, je pensais que je ne m'y habituerais jamais, parce qu'on sent en permanence un tremblement dans ses jambes. Et aussi, si on va quelque part et 
qu'on s'assoit dans le train, et que le train ou le bus roule sur une bosse, on le ressent [une stimulation plus forte]. [..] Mais, à un certain moment, on ne le sent plus. ... Un jour, je me suis assise et j'ai pensé : « Ah, oui, cette chose est toujours en marche, je ne l'ai pas sentie du tout aujourd'hui ».

Si la technologie et les nouvelles sensations qu'elle engendre sont au départ déroutantes et dérangeantes, elles deviennent progressivement incarnées aux niveaux sensoriel et cinétique : la technologie est « faite corps ». Toutefois, l'incarnation de la technologie - le fait qu'elle n'absorbe plus l'attention de son utilisateur/trice - n'explique pas les appréciations contraires exposées plus haut de M. van Houten et Mme Bloemen envers la stimulation de la moelle épinière. Pour que cette technologie soit positivement vécue, elle doit aussi être incorporée, c.-à-d. conçue et vécue comme étant une partie de son corps.

\section{De l'incorporation au « corps en pièces », la question du vieillissement}

Alors que M. van Houten ne tare pas d'éloges sur la stimulation de la moelle épinière, elle est l'objet de souffrances pour Mme Bloemen. Pour eux comme pour toutes les personnes interrogées, la technologie est devenue incarnée. Néanmoins, si M. van Houten la conçoit comme faisant partie de son corps, Mme Bloemen la rejette. Ce qui est en jeu ici est la capacité et la possibilité de s'identifier non seulement visuellement mais aussi tactilement et affectivement avec son corps technologiquement transformé, une identification qui ne se fait pas seul/e mais avec et même par le corps des autres : dans une certaine mesure, pour être incorporée - vécue comme faisant partie de son corps - la technologie se doit d'être incorporée par d'autres (Slatman et Widdershoven, 2010).

À cet égard, son petit-fils et le regard de ce dernier jouent un rôle clé dans l'acceptation par Mme Baten (61 ans) de son corps technologiquement transformé. Comme elle l'explique, son petit-fils aîné 
qui a maintenant 13 ans est au courant [qu'elle vit avec une telle technologie]. Il l'a même vue et pense que c'est ok. ... Il a fait partie du processus [d'implantation] à l'époque, il avait alors 10 ans, il était présent. Et au début il y a jeté un coup d'œil, et c'est très intéressant tout ça, mais après l'avoir vue [..] c'est bon [..] ça fait partie de grand-mère. C'est comme ça.

Le regard approbateur de son petit-fils facilite, et peut-être même permet à Mme Baten de concevoir la stimulation de la moelle épinière comme faisant partie d'elle-même, de son corps. Mme Baten comme M. van Houten s'identifient (affectivement, visuellement et tactilement) à la technologie de neuromodulation - M. van Houten aimerait pouvoir montrer au monde la technologie qui lui permet de prendre soin de son mari.

Quant au rejet de Mme Bloemen envers la stimulation de la moelle épinière, de la même manière, il ne peut pas être compris en dehors de ses relations avec son mari et son fils :

Mme Bloemen (MB) : Je ne voulais pas (de) ça, je ne voulais pas de quelque chose dans mon corps qui n'y a pas sa place. ... La télécommande, on peut la mettre de côté et elle disparaît ! [rires] On n'a pas à y penser. Mais ça c'est, oui, c'est toujours là. On ne peut pas l'enlever.

Auteur : Et, donc cette technologie de neuromodulation, vous ne la considérez pas comme faisant partie de vous?

MB : Oh non ! Non ! C'est d'une grande aide pour moi. Mais ça reste un instrument, et mmh [..] [larmes] Je n'aime pas que [..] qu'il [son mari] puisse le toucher. [..] Oui, au début, on en rie, mais à un certain moment ce n'est plus drôle. Mari de MB : Non, mais pour moi, c'est plus facile de voir [la technologie] comme faisant partie d'elle car sans ça elle ne fonctionne pas très bien. C'est peut-être plus facile pour le conjoint de s'y habituer que pour la personne ellemême. 
Physicienne : Parce que pour vous [MB], il s'agit plus d'un objet pénible ou agaçant que ...

MB : Oui, une chose pénible et parfois encombrante.

Mari de MB : Les seules fois où je le vois, c'est quand on est au lit, pour le dire franchement.

MB : Oui, oui, je sais bien. [..] Mais avec les vêtements, tu dois aussi prendre en compte [la technologie]. Et [..] tu veux être sûre que [..] les autres ne la voient pas.

Bien que Mme Bloemen ait besoin de cette technologie pour « fonctionner », comme le dit son mari, et pour ne plus vivre dans la douleur, elle ne l'accepte pas - celle-ci « n'a pas sa place » dans son corps. Visuellement, tactilement et affectivement, elle ne peut s'identifier à cette technologie ni aux transformations corporelles qu'elle engendre. Dans la mesure où Mme Bloemen ne supporte pas le fait que son mari puisse voir et toucher le neurostimulateur (placé sous la peau), sa non-acceptation de la technologie et sa non-identification avec son corps technologiquement transformé sont intimement liées au corps de son mari - son regard, son toucher. Par ailleurs, un peu plus tôt, lorsqu'elle commença à nous parler de la technologie de neuromodulation, Mme Bloemen fit référence au corps de son fils dans lequel est implantée une pompe à insuline et pour laquelle elle se sent coupable, le diabète de son fils étant génétique.

L'incorporation de la technologie de neuromodulation qui agit sur le corps est entremêlée au corps des autres et souligne à quel point la corporalité - technologiquement transformée ou non - est toujours inter-corporalité. Par ailleurs, l'incorporation n'est pas seulement liée au corps d'êtres aimés et proches mais aussi au regard des autres et son intériorisation. Le rejet voire la révulsion de Mme Bloemen vis-à-vis de la technologie sont 
aussi étroitement liés à ces derniers. La présence de la technologie ne lui permet pas de se vêtir comme elle l'entend, ou plutôt, son corps technologiquement transformé ne lui permet pas de remplir les injonctions sociales - telles qu'elle sont projetées et intériorisées - liées à son âge (45 ans) et son genre, à savoir être valide, en forme («dans la force de l'âge ») et féminine.

Alors que tous les corps sont doubles - ils sont vus parce qu'ils voient, touchés parce qu'ils touchent (Merleau-Ponty, 1945) - la détresse de Mme Bloemen liée à la visibilité du neurostimulateur sous sa peau s'inscrit dans la dimension spéculaire du corps féminin, le corps des femmes étant plus soumis (que celui des hommes) au regard des autres (Bartky, 1997). La présence du neurostimulateur qui bien qu'implanté demeure visible et palpable l'empêche de se conformer à la féminité blanche hétérosexuelle - la féminité hégémonique dont les contours du corps se doivent d'être parfaitement lisses : « la peau d'une femme doit être douce, souple, glabre et lisse ; idéalement, elle ne doit trahir aucun signe d'usure, d'expérience, d'âge ou de pensée profonde » (Ibid., p. 137). La technologie perturbe et empêche réalisation des normes de féminité. Si ce trouble des normes de genre peut être considéré comme bienvenu (Butler, 1990), dans le cas de Mme Bloemen, il est vécu comme un échec.

Le corps transformé par la stimulation de la moelle épinière est un corps vieillissant, voire même déjà vieux et associé à l'invalidité. En fait, cette technologie produit (et atteste d') un corps vieillissant, lequel prend une forme particulière :

Je fais des blagues à propos de ça [cette technologie], je suis très détendue à ce sujet parce que mon dos est cassé, mes yeux [..] je viens d'avoir un détachement de l'iris, j'ai des rhumatismes, j'ai tout un tas de choses [..] je perds mes cheveux, j'ai des implants dans la bouche [..] La seule chose qui manque, c'est une jambe de bois ! » (Mme Baten). 
Les propos de Mme Jansen font écho à ceux de Mme Baten :

Au début, je trouvais l'idée un peu folle, moi tel un robot avec toutes sortes de pièces qu'on remplace.

Auteur : Oui, vous vous sentez comme ça?

MJ : Oui [..] Deux nouveaux yeux [greffes de la cornée] et cette chose dans mon dos et mon ventre [..] Disons [..] Parfois, je pense qu'une paire de nouveaux genoux, ce serait bien ! [rires]

Se concevoir (et s'accepter) comme étant un corps vieillissant (ou vieux) semble aller de pair avec se concevoir comme un « corps en pièces » (Hacking, 2007), lesquelles ne sont peut-être pas détachables mais sont en tout cas remplaçables. Cette identification semble faciliter le processus d'identification avec son corps technologiquement transformé. Portée par les technosciences, cette conception « néo-cartésienne » (Ibid.) change la relation au corps et apparaît comme symptomatique du vieillissement : la présence croissante de technologies dans ou au plus près du corps va de pair avec le vieillissement, le constituant même (Ihde, 2008 ; Macia et al., 2013). Inversement, le vieillissement normalise ou naturalise la présence intime de technologies telles que la stimulation de la moelle épinière. Dans ce contexte, on peut mieux comprendre le rejet exprimé par Mme Bloemen vis-à-vis de la technologie de neuromodulation. Elle n'a après tout que 45 ans et deux enfants (16 et 20 ans) qui vivent à la maison. Le vieillissement et ses effets ne sont pas encore censés être vécus aussi intensément. Comme l'explique Ihde (2008), « l'accumulation progressive des hybridations entre humains et technologies, ou le processus cyborg, est souvent lié aux effets contemporains du vieillissement. ... [L] es stratégies « cyborg » [qui] sont souvent des tentatives technologiques visant à contrecarrer les effets encore plus sévères du vieillissement ... restent des compromis. Il est préférable d'avoir un pacemaker que d'avoir une arythmie mortelle ; il est préférable ... de marcher avec un implant en acier téflon ou une prothèse que de ne pas 
marcher du tout ... Au final, tous ces compromis sont loin des techno-fantasmes bioniques si souvent projetés dans la culture populaire » (p. 38-39). Durant notre entretien, M. Koopman (53 ans) se qualifie d' « homme bionique » lorsqu'il se rappelle de sa sortie de l'hôpital après l'implantation de la technologie de neuromodulation, mais presqu'instantanément il retire ou met à distance cette association : la vulnérabilité de son corps nécessitant une telle technologie et récupérant de la chirurgie ne s'inscrivent pas facilement dans l'imagerie bionique ou cyborg qui est emplie de corps jeunes et musclés que la technologie vient sublimer (Dalibert, 2015 ; Oudshoorn, 2015). Le poids de cet imaginaire complique le processus d'incorporation et d'identification avec son corps technologiquement transformé (Joyce et Mamo, 2006). La vieillesse, bien que clé pour accepter ces technologies et les comprendre, n'a pas sa place dans cet imaginaire qui entoure les technologies transformant les corps. Or, c'est bien le corps vieillissant ou vieux qui est construit par les technologies « bioniques » de neuromodulation. Ce corps est « un corps en pièces ».

\section{Conclusion}

Implantée, la stimulation de la moelle épinière demande un processus d'apprentissage - un tâtonnement - au cours duquel le corps est mobilisé : les gestes et les postures sont essentiels à l'incarnation de la technologie, c.-à-d. pour qu'elle soit vécue de manière transparente. Cette technologie crée de nouvelles sensations et transforme le corps. Être capable de s’identifier à ce corps technologiquement modifié est nécessaire pour que la technologie soit vécue de manière satisfaisante. Dans ce processus visuel, tactile et affectif, le corps des autres, proches ou lointains, aimés ou inconnus, est central. Qu'il s'agisse du regard approbateur d'un petitfils ou de la réalisation que celui que l'on aime puisse voir et toucher le corps que l'on n'accepte pas, l'autre est partie intégrante du processus d'incorporation. Les normes sociales y sont aussi déterminantes dans la mesure où la présence de la technologie peut être vécue 
comme un obstacle - et être synonyme d'échec - pour être une femme féminine ou un homme dont la masculinité correspond à celle de l'imaginaire et l'imagerie cyborgs et bioniques. À l'inverse, se concevoir comme vieillissant facilite le processus d'incorporation et apparait même comme une condition nécessaire au bien vivre avec une technologie de neuromodulation. Peu appréhendé comme allant de pair avec les technologies « cyborgs », le vieillissement est clé pour pouvoir bien vivre avec des technologies implantées telles que la stimulation de la moelle épinière. Alors qu'il n'y a dans nos sociétés ni rite de passage ni âge précis qui marque l'entrée dans la vieillesse, il semble que l'intimité croissante avec les technologies qui touchent au corps constitue le corps vieillissant. Il se fait corps en pièces.

\section{Références :}

Akrich, M. (1992). The De-scription of Technical Objects. Dans W. Bijker et J. Law (dir.) Shaping Technology/Building Society : Studies in Sociotechnical Change (205-24). Cambridge : The MIT Press.

Bartky, S. (1997). Foucault, Femininity, and the Modernization of Patriarchal Power. Dans K. Conboy, N. Medina et S. Stanbury (dir.) Writing on the Body : Female Embodiment and Feminist Theory (128-154). New York : Columbia University Press.

Betcher, S. (2001). Putting My Foot (Prosthesis, Crutches, Phantom) Down : Considering Technology as Transcendence in the Writings of Donna Haraway. Women's Studies Quarterly, 29(3-4), 35-53.

Butler, J. (1990). Gender Trouble : Feminism and the Subversion of Identity. New York : Routledge.

Dalibert, L. (2014). Posthumanism and Somatechnologies : Exploring the Intimate Relations between Humans and Technologies. Thèse de doctorat (Université de Twente, Enschede, Pays-Bas). 
Dalibert, L. (2015). Remarquables mais non (re-)marqués : Le rôle du genre et de la blanchité dans les représentations des corps « technologisés ». Poli, 10, 50-59.

De Preester, H. (2011). Technology and the Body : The (Im)Posssibilities of Re-embodiment. Foundations of Science, 16(2), 119-37.

Estes, C.L. et Binney, E.A. (1989). The Biomedicalization of Aging : Dangers and Dilemmas. The Gerontologist, 29(5), 587-96.

Gérardin, P. et Andrieu, A. (2011). La continuité de soi : Vers une hybridité créative. Gérontologie et société, 137 (2), 151-62.

Hacking, I. (2007). Our Neo-Cartesian Bodies in Parts. Critical Inquiry, 34(1), 78-105.

Haraway, D. (1991). Simians, Cyborgs, and Women: The Reinvention of Nature. Londres : Free Association Books.

Ihde, D. (1990). Technology and the Lifeworld : From Garden to Earth. Bloomington : Indiana University Press.

Ihde, D. (2008). Aging : I Don't Want to Be a Cyborg! Phenomenology \& the Cognitive Sciences, 7(3), 397-404.

Joyce, K. et Loe, M. (2010). A Sociological Approach to Ageing, Technology and Health. Sociology of Health \& Illness, 32(2), 171-80.

Joyce, K. et Mamo, L. (2006). Graying the Cyborg : New Directions of Feminist Analyses of Aging, Science, and Technology. Dans T. Calasanti and K. Slevin (dir.) Age Matters : Realigning Feminist Thinking (99-121). New York : Routledge.

Kaufman, S.R., Shim, J.K. et Russ, A.J. (2004). Revisiting the Biomedicalization of Aging : Clinical Trends and Ethical Challenges. The Gerontologist, 44(6), 731-8.

Kaufman, S.R., Mueller, P.S., Ottenberg, A.L. et Koenig, B.A. (2011). Ironic Technology : Old Age and the Implantable Cardioverter Defibrillator in US Health Care. Social Science \& Medicine, 72(1), 6-14. 
Latour, B. (2009 [1992]). Where Are the Missing Masses? The Sociology of a Few Mundane Artifacts. Dans D. G. Johnson et J. M. Wetmore (dir.) Technology and Society: Building our Sociotechnical Future (151-80). Cambridge : The MIT Press.

Laz, C. (2003). Age Embodied. Journal of Aging Studies, 17(4), 503-19.

Macia, E., Duboz, P. et Chevé, D. (2013). La fin de la dépendance ? Transformations contemporaines des corps-sujets vieillissants. Gérontologie et société, 145(2), 167-77.

Maestrutti, M. (2011). Cyborg Identities and Contemporary Techno-Utopias Adaptations and Transformations of the Body in the Age of Nanotechnology. Journal international de bioéthique, 22(1-2), 71-88.

Merleau-Ponty, M. (1945). Phénoménologie de la perception. Paris : Gallimard.

Mort, M., Robert, C. and Callén, B. (2013). Ageing with Telecare : Care and Coercion in Austerity? Sociology of Health \& Illness, 35(6), 799-812.

Neven, L. (2011). Representations of the Old and Ageing in the Design of the New and Emerging : Assessing the Design of Ambient Intelligence Technologies for Older People. Thèse de doctorat (Université de Twente, Enschede, Pays-Bas).

Noland, C. (2009). Agency and Embodiment : Performing Gestures/Producing Culture. Harvard : Harvard University Press.

Oudshoorn, N. (2015). Sustaining Cyborgs : Sensing and Tuning Agencies of Pacemakers and Implantable Cardioverter Defibrillators. Social Studies of Science, 45(1), 56-76.

Oudshoorn, N. et Pinch, T. (2003). How Users Matter : The Co-Construction of Users and Technology. Cambridge : The MIT Press.

Peine, A., Faulkner, A., Jæger, B. et Moors, E. (2015). Science, Technology and the "Grand Challenge" of Ageing - Understanding the Socio-Material Constitution of Later Life. Technological Forecasting \& Social Change, 93, 1-9.

Slatman, J. et Widdershoven, G. (2010). Hand Transplant and Bodily Integrity. Body \& 
Society, 16(3), 69-92.

Sobchack, V. (2006). A Leg to Stand On : On Prosthetics, Metaphor, and Materiality. Dans M. Smith et J. Morra (dir.) The Prosthetic Impulse : From a Posthuman Present to a Biocultural Future (17-41). Cambridge : The MIT Press.

Verbeek, P-P. (2005). What Things Do : Philosophical Reflections on Technology, Agency, and Design, University Park : The Pennsylvania State University. 